\title{
The Application of Information Technology in Science and Technology English Translation
}

\author{
Yonglan LI \\ Guizhou University of Finance\&Economics, Guiyang,Guizhou, 550025,China \\ YonglanLi@yeah.net
}

Keywords: Scientific English; translation IT

\begin{abstract}
Currently, scientific English has become a very important English language body. The translation of scientific English is different from others. Technology English translation must follow certain translation methods and skills. This article focuses on business user experience management from the aspects of translation software, network resources and search engine and detailed the analysis of the application of information technology in the functions of scientific English translation, which will play a pivotal role.
\end{abstract}

\section{Introduction}

English for Science and Technology, abbreviated EST, as an important English style, has the following characteristics compared with non-tech English: declaring things, strong logic, highly structured, variety of terms, rigorous language and accurate data. Technical English generally refers to the English used in natural science and engineering technology. With the development of science and technology and globalization, science and technology English is widely used in science and technology books, papers and lectures to promote international academic exchanges, so technology more and more highlights the importance of international English communication.

Currently, IT has rapidly developed and has been widely used in various fields of production, life and so on. What applications of IT disciplines in science and technology English translation have? How can we effectively use information technology solve problems in IT English translation and thus improve the efficiency of translation? First, we can be supported by translation software. Though there are more or less various defects of the application of translation software in scientific English translation. While because of its early start and highly specialty in many translation tools, translation software is our preferred information translation tools. Secondly, in scientific English translation process, make full use of network resources, its convenience and real-time are fit for the translation of new vocabulary and is indispensable information assisted translation tools. Finally, with the widely application of various search engines, we can combine translation function of various search engines to preliminary understand the information contained in scientific English and help filter our information.

This paper combines various recent IT to explore its applications in English translation to provide a good reference for the majority of translators.

\section{Translation software assistive technology English translation}

\section{Translation software status quo}

In recent years, the domestic translation software achieve the transition from DOS to W inflow s platform, there has been an unprecedented excellent situation. New products continue to emerge, the old product rejuvenated. In the fierce competition of the market, many productions have been eliminated, such as the old translation softwares Landau, Yilin seem to have been uninterested, weir flag bearing drums. Currently, the popular translation software on the domestic market are: interpreter, Masanobu, Hanshin, translated king, Transtar, letter translation, instant Chinese experts, Instant Messenger, the Orient Express, Kingsoft, the global village and the Southern Cross, etc., and also a few foreign translation software to compete. 


\section{Translation software development trends}

Translation software has the following aspects of developing trends: First, specialization. Translation software should clear their translation field, which can greatly improve the translation accuracy. Next is intelligence, which should have the translation memory function and functions of accurately, automatically, repeatedly translate the similar paragraph. In short, professional and intelligent development trend of translation software is benefit for the use of science and technology English translation.

\section{Technology English translation software}

Although the quality of machine translation is still poor, but it can also bring a lot of convenience to translation work with the rational use and improve translation efficiency. Depending on the translation task can be considered from the following aspects: First, the appropriate choice of translation software. Translation software available in the market is complex and diverse and feature has different emphases. Technology English translation areas need professional translation software, for example, select Trados to assist Technology English translation. Trados is the first software to introduce the translation memory function, which feature is a core function of computer-assisted translation. Second, focus on the establishment of a personalized thesaurus and we should constantly enrich and improve the science and technology English related thesaurus in the translation process.

\section{Network resources assistive technology English translation}

Translator of the information age not only should have good command of English and Chinese level and should also have the ability to use the Internet to acquire, store, retrieve information and the function of retrieval and counseling. We can encourage students to read and translate the novelty contents and moderate difficulty professional journal literature from periodicals like Science, Nature and so on, which makes up for the lack of teaching materials and also train the students' ability to initiatively find information and comprehensively analyze the material.

English and Chinese law because of the different rhetoric, different traditional style, different social backgrounds, different ways of thinking and performance practices, etc., some sentences can not be understood just relying on grammatical relationships and must also judge from a logical sense or professional content [2]. Therefore, the use of network resources to assist technology English translation is a shortcut to solve these problems.

Find related pages and be familiar with the the subject areas of original text. When coming into contact with the article needed to be translated, the translator probably did not totally understand the subject areas. So, to be an excellent translators, a standard is that know ABC of everything. Contents on the Internet cover a wide disciplines and can often find the same discipline pages, lucky, you can also find the discipline of English vocabulary.

For example, some character background knowledge, a variety of appliances and equipment items are available at Wikipedia (http: //zh.wikipedia.Org/wiki/). Some historical news background can find the report of authoritative news media (eg New York Times: http: //www.nytimes.com) through the network and understand the news background and makes us more accurately translate of the text original meaning and the author's stand point of view. 


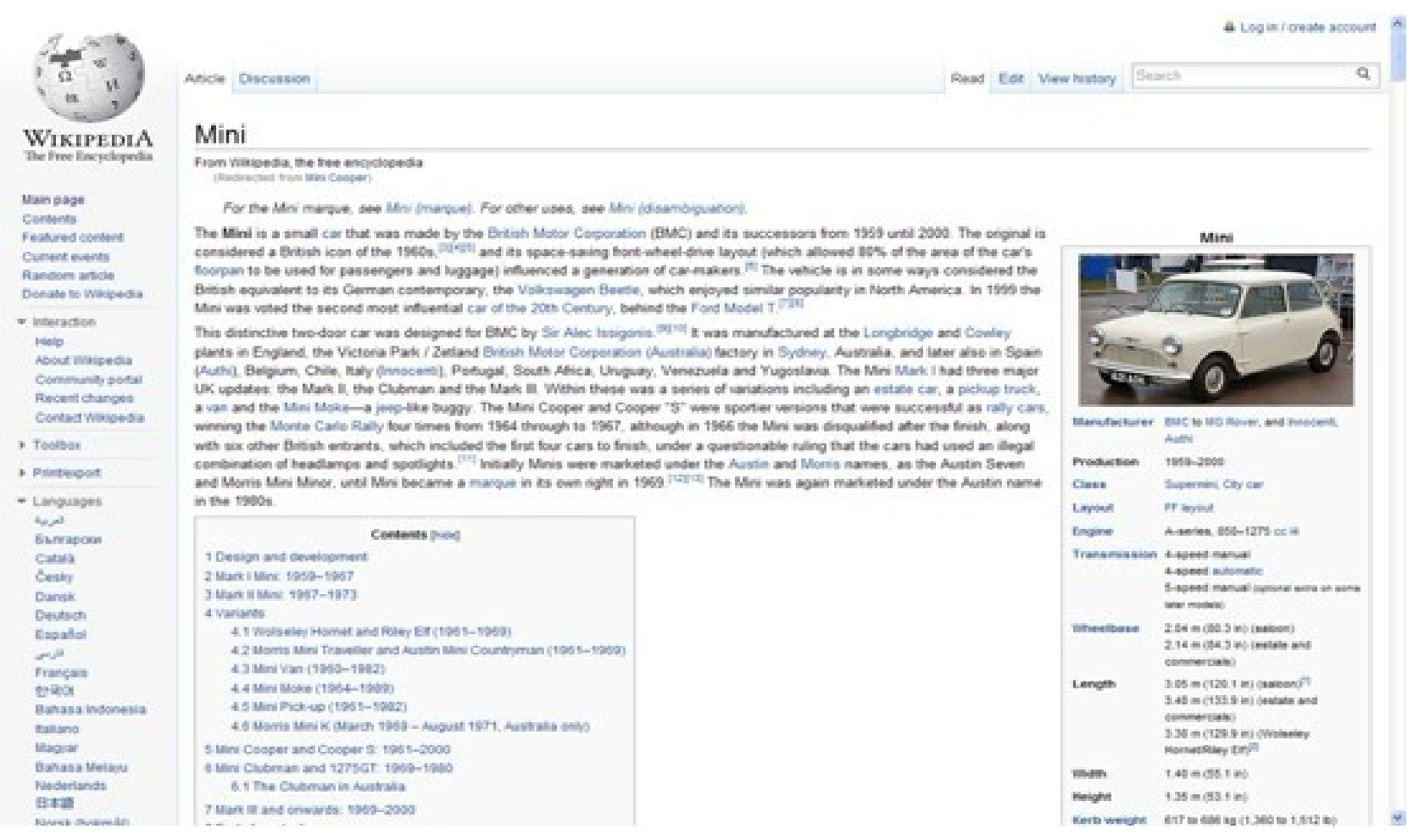

Figure 1 Kiwi Wikipedia web interface

\section{Search engines assistive technology English translation}

A lot of new vocabulary appears in the translation of technology English because of huge different English and Chinese characters, which cannot all be timely included in the traditional dictionary. Thus the understanding and translation of the vocabulary continuous development often make translators feel hard. In fact, the use of search engines can easily find a more accurate translation of the vocabulary.

Using the search engine aided translation can overcome text comprehension before translation and expression of words in the translation, or check and proofread after the translation. The Internet can provide great help for translators. The Internet is equivalent of a free giant corpus and search engine is the key to open this corpus. Make full use of the search engine to assist us in scientific and technology English translation to quickly retrieval expertise knowledge we need.

\section{Search engine-assisted English translation}

Development of the Internet is closely related with the development of search engine. In scientific English translation, the search engine can also help us translate, for example, to improve the translation efficiency and accuracy of technology English referring to the existing translation literature, especially some new vocabulary not collected in the dictionary and to find some specific meaning of some acronyms. Here are several methods of its use.

Suppose that we do not know how to translate netizens the word, but based on the experience we infer that the word may contain the letter combinations of net, then, so we type net users to search in the common engines (eg Google: www.google com, MSN Search:. Http: // search. msn. com). We can find the the correct translation in the searching results. Some words with Chinese characteristics is the difficulty when we translate. Those words often are not included in the traditional dictionary, but a lot of literature in translation have a convention, so we can refer to its translation. Here we take the league secretary for example, in the translation we may not know specifically how to translate it, but we can speculate that it is likely to contain league, so we type league secretary in the search engine to search, and got the results you want in the first line of the first page : League branch secretary.

Google also launched the Google Online Translator, which support for translation among languages and support Web page translation and simply enter the URL needed to be translated. 
Currently, many search engine with translation function frequently come out. We can forecast the future Internet search engine has not merely translation tools of translators but the translation means they must master.

Therefore, the advantages of the use of search engines assist technology English translation are obvious. To keep the advance of the time, translators should strive to improve their professional quality and constantly sum up in practice to make their works more perfect.

\section{Conclusion}

Technical English doesn't have distinct emotional, perceptual thinking like ordinary English and its purpose is for readers to easily understand the reader and accurately express the objective laws, according to logical thinking, articulately state the problem. With the accelerated development of the globalization process, the importance of science and technology English translation is constantly highlighted. But science and technology English from the content and form will continue to enrich and update, while the development of information technology resources conform to the way people use the Internet and search engine technology to improve the English translation requirements. Meanwhile, in order to shorten the translation process and improve translation efficiency, it is needed that translation software continue to develop towards a more Intelligent direction.

\section{References}

[1] Luo Xuanmin. Chinese Translation Teaching: Problems and Prospects[J] Chinese Translation, 2003, (4): 56 - 58.

[2] National Higher University Foreign Language Teaching and Research. Seminar on University English teaching reformation [J]. Foreign Language, 2003, (2): 7 - 11

[3] Li Sheng. The Application of Translation Variation in Scientific English Translation [D]. Hunan Normal University,2013.

[4] Wang Yuhang. The application of Schema Theory in Scientific English Translation[D].Ha Erbin Science and Engneering University, 2010.

[5] Wan Lihua. Study on the English Translation skills of Translating Scientific English into Chinese[D]. The Central China Normal University, 2014. 